\title{
UNCONDITIONAL BASES IN TENSOR PRODUCTS OF HILBERT SPACES
}

\author{
DAVID PÉREZ-GARCÍA and IGNACIO VILLANUEVA*
}

\begin{abstract}
We prove that a tensor norm $\alpha$ (defined on tensor products of Hilbert spaces) is the Hilbert-Schmidt norm if and only if $\ell_{2} \otimes \cdots \otimes \ell_{2}$, endowed with the norm $\alpha$, has an unconditional basis. This extends a classical result of Kwapień and Pełczyński. The symmetric version of that statement follows, and this extends a recent result of Defant, Díaz, García and Maestre.
\end{abstract}

\section{Introduction}

In [16], Kwapień and Pełczyński proved that if we have a tensor norm $\alpha$ defined on tensor products of Hilbert spaces such that $\ell_{2} \hat{\otimes}_{\alpha} \ell_{2}$ has an unconditional basis, then $\alpha$ has to be (equivalent to) the Hilbert-Schmidt norm. Their technique involves the Schmidt decomposition of a compact bilinear form from Hilbert spaces. The problem is that, up to now, there is no reasonable analogue of this decomposition for trilinear forms (the only version is the normal form given in [2], which is too complicated to be useful here). This is the reason we need new techniques to extend this result to more than two spaces. Our main result is the following (Theorem 2.5)

THEOREM. If $\alpha$ is a tensor norm (defined on tensor products of Hilbert spaces) such that $\hat{\otimes}_{\alpha, j=1}^{n} \ell_{2}$ has an unconditional basis, then $\alpha$ is equivalent to the Hilbert-Schmidt norm $\sigma_{2}$.

The study of Hilbert-Schmidt operators (or bilinear forms) goes back to the work of Hilbert and Schmidt about integral equations at the beginning of the twentieth century. Since then, they have been constantly applied both in pure and applied mathematics. The generalization of this class to the multilinear setting goes back to Dwyer III [12] and was recovered independently by Pietsch [23] and Janson, Peetre and Rochberg [15], where it is shown how this class can be applied to the study of Hankel forms. Motivated by this application Cobos, Kühn and Peetre continued the study in a series of papers [2], [3], [4],

\footnotetext{
* Partially supported by BMF 2001-1240.

Received June 1, 2004.
} 
[5], where they give a first solution to the study of the Schmidt decomposition of a multilinear form (the normal form). Very recently, Matos [17] gave a new step, relating the Hilbert-Schmidt multilinear operators to the class of multiple summing operators. This work was finished in [19] where it is proved that these two classes are exactly the same, giving a multilinear extension of the main theorem of [18].

In the context of the theory of polynomials in Banach spaces, the problem of finding unconditional bases in symmetric tensor products has attracted recently a lot of attention (see for instance [7] or [10] and the references therein). The symmetric case of Kwapień and Pełczyński's result appeared in [6], only for the case of two spaces. In Theorem 2.6 we extend it to the general case.

Here we will take advantage of the results in [19]. Particularly, the key tool in our proof of the above Theorem will be the following result, proved in [19] (see below for the definitions):

Theorem 1.1. Let $T: \ell_{2} \times \cdots \times \ell_{2} \longrightarrow \mathrm{K}$ be a multilinear form. If $T$ is multiple 1-summing, then $T$ is Hilbert-Schmidt and

$$
\|T\|_{S_{2}} \leq 2^{\frac{n}{2}} \pi_{1}(T) .
$$

The theory of multiple summing multilinear operators has been recently developed by Bombal and both authors in [1], [19], [20], [21], [22], and by Matos in [17], where it is shown how this class properly generalizes the linear behavior of $p$-summing operators. This paper represents then another application of this theory to the study of the structure of tensor products (other applications can be found in the above references).

We remind the reader that, for a finite sequence $\left(x_{i}\right)_{i=1}^{m} \subset X$ and $1 \leq p<$ $\infty$, we will write $\left\|\left(x_{i}\right)_{i=1}^{m}\right\|_{p}^{\omega}$ to denote

$$
\sup \left\{\left(\sum_{i=1}^{m}\left|x^{*}\left(x_{i}\right)\right|^{p}\right)^{\frac{1}{p}}: x^{*} \in B_{X^{*}}\right\} .
$$

Definition 1.2. Let $1 \leq p<+\infty$. A multilinear operator $T: X_{1} \times \cdots \times$ $X_{n} \longrightarrow Y$ is multiple $p$-summing if there exists a constant $K>0$ such that, for every choice of sequences $\left(x_{i_{j}}^{j}\right)_{i_{j}=1}^{m_{j}} \subset X_{j}$, the following relation holds

$$
\left(\sum_{i_{1}, \ldots, i_{n}=1}^{m_{1}, \ldots, m_{n}}\left\|T\left(x_{i_{1}}^{1}, \ldots, x_{i_{n}}^{n}\right)\right\|^{p}\right)^{\frac{1}{p}} \leq K \prod_{j=1}^{n}\left\|\left(x_{i_{j}}^{j}\right)_{i_{j}=1}^{m_{j}}\right\|_{p}^{\omega} .
$$

In that case, we define the multiple p-summing norm of $T$ by $\pi_{p}(T)=$ $\min \{K: K$ verifies (1) $\}$ 
We will use the following straightforward characterization of multiple 1summing operators

LemMa 1.3. A multilinear operator $T: X_{1} \times \cdots \times X_{n} \longrightarrow Y$ is multiple 1summing if and only if there exists a constant $K$ such that for every $1 \leq j \leq n$, for every $m_{j} \in \mathrm{N}$ and for every $u_{j}: \ell_{\infty}^{m_{j}} \longrightarrow X_{j}$ with $\left\|u_{j}\right\| \leq 1$, we have that

$$
\sum_{i_{1}, \ldots, i_{m}=1}^{m_{1}, \ldots, m_{n}}\left|T\left(u_{1}\left(e_{i_{1}}\right), \ldots, u_{n}\left(e_{i_{n}}\right)\right)\right| \leq K
$$

where $\left(e_{i_{j}}\right)_{i_{j}=1}^{m_{j}}$ is the canonical basis in $\ell_{\infty}^{m_{j}}$. In that case we have that $\pi_{1}(T)$ is just the infimum of such constants $K$.

The notation will be the standard in the context and [9], [11] will be our basic references for unexplained notation.

All along this paper all the operators are supposed to be continuous. Given $X, Y$ Banach spaces, $\mathscr{L}(X, Y)$ will denote the Banach space of linear (and continuous) operators. $B_{X}$ will be the closed unit ball of the Banach space $X$ and $\mathrm{K}$ will denote the scalar field, either $\mathrm{R}$ or $\mathrm{C}$. If $\lambda \in \mathrm{K}^{k}$, we write $d_{\lambda}: \mathrm{K}^{k} \longrightarrow \mathrm{K}^{k}$ for the diagonal operator $d_{\lambda}(x)=\left(\lambda_{i} x_{i}\right)_{i=1}^{k}$.

Let $X$ be a Banach space and let $I$ be either $\mathrm{N}$ or $\{1, \ldots, m\}$. A sequence $\left(x_{i}\right)_{i \in I}$ of non-zero vectors is an unconditional basic sequence in $X$ if there is a constant $K$ such that

$$
\left\|\sum_{i \in I} \epsilon_{i} \mu_{i} x_{i}\right\| \leq K\left\|\sum_{i \in I} \mu_{i} x_{i}\right\|
$$

for every $\epsilon_{i}, \mu_{i} \in \mathrm{K}$ with $\left|\epsilon_{i}\right| \leq 1$. The best of such constants $K$ is called the unconditional constant of $\left(x_{i}\right)_{i \in I}$. If a Banach space $X$ has an unconditional basis, we define $u b(X)$ as the infimum of the unconditional constants of all the unconditional basis of $X$.

In this paper, we will deal with tensor norms of order $n$ defined on tensor products of Hilbert spaces. We will call them simply tensor norms; that is, for us, a tensor norm $\alpha$ will be a method of ascribing to each choice $H_{1}, H_{2}, \ldots, H_{n}$ of $n$ Hilbert spaces, a norm (that we will also call $\alpha$ ) in the tensor product $H_{1} \otimes \cdots \otimes H_{n}$ such that

i) $\epsilon \leq \alpha \leq \pi$, where $\epsilon$ and $\pi$ are, respectively, the injective and projective norms.

ii) For every $u_{j} \in \mathscr{L}\left(H_{j}, K_{j}\right)(1 \leq j \leq n)$, where $H_{j}, K_{j}$ are Hilbert spaces for $1 \leq j \leq n$, we have that $u_{1} \otimes \cdots \otimes u_{n}$ is $\alpha-\alpha$ continuous with norm less or equal than $\prod_{j=1}^{n}\left\|u_{j}\right\|$. 
We will write $\otimes_{\alpha, j=1}^{n} H_{j}$ for the tensor product endowed with the norm $\alpha$, and $\hat{\otimes}_{\alpha, j=1}^{n} H_{j}$ for its completion.

We do not know of any general reference for tensor norms of order $n$, though one can find the definition and some properties in [14]. However, everything we are going to use is a straightforward generalization of the case $n=2$, for which we refer to [9] (specially to [9, Chapter 26], where the case of tensor norms defined on Hilbert spaces is treated). If $\alpha$ is a tensor norm, $\alpha^{\prime}$ will be its dual tensor norm.

We will need the following result that appeared in [7, Remark 1] (see [24] and [25] for the case of two spaces). We write it in the case we are going to use.

THEOREM 1.4. If $\alpha$ is a tensor norm, we have that $\hat{\otimes}_{\alpha, j=1}^{n} \ell_{2}$ has an unconditional basis if and only if $f_{i_{1}}^{1} \otimes \cdots \otimes f_{i_{n}}^{n}$ is an unconditional basis in $\hat{\otimes}_{\alpha, j=1}^{n} \ell_{2}$ for any choice of orthonormal basis $\left(f_{i_{j}}^{j}\right)_{i_{j}=1}^{\infty} \subset \ell_{2}$. In fact, in that case, the unconditional constant of $f_{i_{1}}^{1} \otimes \cdots \otimes f_{i_{n}}^{n}$ is bounded by

$$
2^{n+1} \mathrm{ub}\left(\hat{\otimes}_{\alpha, j=1}^{n} \ell_{2}\right) \text {. }
$$

Finally, we recall the definition of the Hilbert-Schmidt norm $\sigma_{2}$.

Definition 1.5. A multilinear form $T: H_{1} \times \cdots \times H_{n} \longrightarrow \mathrm{K}$ defined on Hilbert spaces is Hilbert-Schmidt if

$$
\left(\sum_{i_{1} \in I_{1}, \ldots, i_{n} \in I_{n}}\left|T\left(e_{i_{1}}^{1}, \ldots, e_{i_{n}}^{n}\right)\right|^{2}\right)^{\frac{1}{2}}<\infty,
$$

where $\left(e_{i_{j}}^{j}\right)_{i_{j} \in I_{j}} \subset H_{j}$ is an orthonormal basis $(1 \leq j \leq n)$. It is easy to see that the expression (2) does not depend on the choice of the orthonormal bases [17, Proposición 5.1]. In that case, (2) is the Hilbert-Schmidt norm of $T$ and we will denote it by $\|T\|_{S_{2}}$. The class of Hilbert-Schmidt forms is denoted by $S_{2}\left(H_{1}, \ldots, H_{n}\right)$.

Now (see [17]) one can see the Hilbert-Schmidt norm $\sigma_{2}$ as the tensor norm that satisfies

$$
\left(\hat{\otimes}_{\sigma_{2}, j=1}^{n} H_{j}\right)^{*}=S_{2}\left(H_{1}, \ldots, H_{n}\right) \text {. }
$$

\section{The Result}

We need first some lemmas.

LEMMA 2.1. If $k \leq m$, each $u$ in the unit ball of $\mathscr{L}\left(\ell_{2}^{k}, \ell_{2}^{m}\right)$ can be written as a convex combination of isometries. As a consequence, if $k \in \mathrm{N}$, each $u$ 
in the unit ball of $\mathscr{L}\left(\ell_{2}^{k}, \ell_{2}\right)$ can also be written as a convex combination of isometries.

Proof. By Krein-Milman's Theorem, it is enough to show that every extreme point of the unit ball of $\mathscr{L}\left(\ell_{2}^{k}, \ell_{2}^{m}\right)$ is an isometry.

Let $u: \ell_{2}^{k} \longrightarrow \ell_{2}^{m}$ be with $\|u\|=1$. We consider its Schmidt decomposition [11, Theorem 4.1]

$$
u(\cdot)=\sum_{i=1}^{k} \lambda_{i}\left(\cdot \mid e_{i}\right) f_{i}
$$

where $\left(e_{i}\right)_{i=1}^{k} \subset \ell_{2}^{k},\left(f_{i}\right)_{i=1}^{k} \subset \ell_{2}^{m}$ are orthonormal sequences and

$$
\|u\|=\lambda_{1} \geq \cdots \geq \lambda_{k} .
$$

If $u$ is not an isometry we have that $\lambda_{k}<1$ and then $u$ can be written as a convex combination of

$$
\sum_{i=1}^{k-1} \lambda_{i}\left(\cdot \mid e_{i}\right) f_{i}+\left(\cdot \mid e_{k}\right) f_{k} \quad \text { and } \quad \sum_{i=1}^{k-1} \lambda_{i}\left(\cdot \mid e_{i}\right) f_{i}-\left(\cdot \mid e_{k}\right) f_{k},
$$

which are also in the unit ball of $\mathscr{L}\left(\ell_{2}^{k}, \ell_{2}^{m}\right)$.

Lemma 2.2. If $u: \ell_{\infty}^{k} \longrightarrow \ell_{2}$ satisfies $\|u\| \leq 1$, then we can find an element $\lambda \in B_{\ell_{2}^{k}}$, and a convex combination of isometries $v_{r}: \ell_{2}^{k} \longrightarrow \ell_{2}$, say $\sum_{r=1}^{R} \mu_{r} v_{r}$, such that

$$
u=\pi_{2}(u) \sum_{r=1}^{R} \mu_{r}\left(v_{r} \circ d_{\lambda}\right),
$$

where $\pi_{2}(u)$ is the 2-summing norm of $u$.

Proof. By Pietsch' Factorization Theorem, there exists a regular probability measure $v$ on $\{1, \ldots, k\}$ and an operator $v: L_{2}(v) \longrightarrow \ell_{2}$, with $\|v\|=\pi_{2}(u)$, such that the following diagram commutes

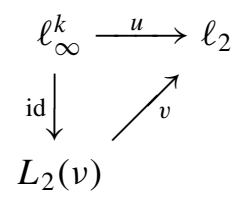

Now, the operator $w: L_{2}(v) \longrightarrow \ell_{2}^{k}$ given by $w(x)=\left(x_{i} \sqrt{v(i)}\right)_{i=1}^{k}$ is an isometry and so we can extend $v$ to $\ell_{2}^{k}$ with the same norm (we still denote 
the extension by $v$ ). Then, if we call $\lambda=(\sqrt{v(i)})_{i=1}^{k} \in B_{\ell_{2}^{k}}$, we obtain the following diagram

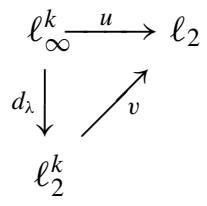

Finally, if we apply Lemma 2.1, we obtain that $\frac{v}{\pi_{2}(u)}$ can be written as a convex combination of isometries $\frac{v}{\pi_{2}(u)}=\sum_{r=1}^{R} \mu_{r} v_{r}$ and we are done.

LEMma 2.3. If $T: \ell_{2} \times \cdots \times \ell_{2} \longrightarrow \mathrm{K}$ is a multilinear form, we have that

$$
\pi_{1}(T) \leq K_{G}^{n} \sup \left\{\sum_{i_{1}, \ldots, i_{n}=1}^{m_{1}, \ldots, m_{n}}\left|\lambda_{i_{1}}^{1} \cdots \lambda_{i_{n}}^{n} T\left(f_{i_{1}}^{1}, \ldots, f_{i_{n}}^{n}\right)\right|\right\}
$$

where $K_{G}$ is Grothendieck's constant and the supremum is taken among all the orthonormal sequences $\left(f_{i_{j}}^{j}\right)_{i_{j}=1}^{m_{j}}$ of $\ell_{2}$ and all the elements $\lambda^{j} \in B_{\ell_{2}^{m_{j}}}$.

Proof. Following Lemma 1.3 we consider an arbitrary $\epsilon>0$ and operators $u_{j}: \ell_{\infty}^{m_{j}} \longrightarrow \ell_{2}$ with $\|u\| \leq 1$ such that

$$
\pi_{1}(T)-\epsilon \leq \sum_{i_{1}, \ldots, i_{n}=1}^{m_{1}, \ldots, m_{n}}\left|T\left(u_{1}\left(e_{i_{1}}\right), \ldots, u_{n}\left(e_{i_{n}}\right)\right)\right|
$$

where $\left(e_{i_{j}}\right)$ denotes the canonical basis of $\ell_{\infty}^{m_{j}}$. Now we use Lemma 2.2 to obtain, for each $1 \leq j \leq n$, an element $\lambda^{j} \in B_{\ell_{2}^{m_{j}}}$ and a convex combination of isometries $\sum_{r_{j}=1}^{R_{j}} \mu_{r_{j}}^{j} v_{r_{j}}^{j}$ such that

$$
u_{j}=\pi_{2}\left(u_{j}\right) \sum_{r_{j}=1}^{R_{j}} \mu_{r_{j}}^{j}\left(v_{r_{j}}^{j} \circ d_{\lambda^{j}}\right)
$$

By Grothendieck's Theorem, $\pi_{2}\left(u_{j}\right) \leq K_{G}$ for every $j$ and therefore, $\pi_{1}(T)-\epsilon \leq \sum_{r_{1}, \ldots, r_{n}=1}^{R_{1}, \ldots, R_{n}} \mu_{r_{1}}^{1} \ldots \mu_{r_{n}}^{n} K_{G}^{n} \sum_{i_{1}, \ldots, i_{n}=1}^{m_{1}, \ldots, m_{n}}\left|\lambda_{i_{1}}^{1} \cdots \lambda_{i_{n}}^{n} T\left(v_{r_{1}}^{1}\left(e_{i_{1}}\right), \ldots, v_{r_{n}}^{n}\left(e_{i_{n}}\right)\right)\right|$.

Now, for each $1 \leq j \leq n$ and each $r_{j}$, we have that $\left(v_{r_{j}}^{j}\left(e_{i_{j}}^{j}\right)\right)_{i_{j}=1}^{m_{j}}$ is an orthonormal sequence in $\ell_{2}$. Then

$$
K_{G}^{n} \sum_{i_{1}, \ldots, i_{n}=1}^{m_{1}, \ldots, m_{n}}\left|\lambda_{i_{1}}^{1} \cdots \lambda_{i_{n}}^{n} T\left(v_{r_{1}}^{1}\left(e_{i_{1}}\right), \ldots, v_{r_{n}}^{n}\left(e_{i_{n}}\right)\right)\right|
$$


is bounded by the right hand side of (3) and we are done.

LeMma 2.4. If $\alpha$ is a tensor norm such that $\hat{\otimes}_{\alpha, j=1}^{n} \ell_{2}$ has an unconditional basis, then we have that

$$
\alpha \leq K \mathrm{ub}\left(\hat{\otimes}_{\alpha, j=1}^{n} \ell_{2}\right) \sigma_{2}
$$

in $\otimes_{j=1}^{n} \ell_{2}$, where $K=2^{\frac{3}{2} n+1} K_{G}^{n}$.

Proof. By Theorem 1.4, for any orthonormal sequence $\left(f_{i_{j}}^{j}\right)_{i_{j}=1}^{m_{j}}$ in $\ell_{2}(1 \leq$ $j \leq n)$, the unconditional constant of $\left(f_{i_{1}}^{1} \otimes \cdots \otimes f_{i_{n}}^{n}\right)_{i_{1}, \ldots, i_{n}=1}^{m_{1}, \ldots, m_{n}}$ in $\hat{\otimes}_{\alpha, j=1}^{n} \ell_{2}$ is bounded by

$$
2^{n+1} \mathrm{ub}\left(\hat{\otimes}_{\alpha, j=1}^{n} \ell_{2}\right) \text {. }
$$

Therefore, if $T: \ell_{2} \times \cdots \times \ell_{2} \longrightarrow \mathrm{K}$ belongs to the unit ball of $\left(\hat{\otimes}_{\alpha, j=1}^{n} \ell_{2}\right)^{*}$, we have that

$$
\sum_{i_{1}, \ldots, i_{n}=1}^{m_{1}, \ldots, m_{n}}\left|\lambda_{i_{1}}^{1} \cdots \lambda_{i_{n}}^{n} T\left(f_{i_{1}}^{1}, \ldots, f_{i_{n}}^{n}\right)\right| \leq 2^{n+1} \mathrm{ub}\left(\hat{\otimes}_{\alpha, j=1}^{n} \ell_{2}\right) \prod_{j=1}^{n}\left\|\sum_{i_{j}=1}^{m_{j}} \lambda_{i_{j}}^{j} f_{i_{j}}^{j}\right\|
$$

for every choice of scalars $\lambda_{i_{j}}^{j}$. Therefore, Lemma 2.3 tells us that $T$ is multiple 1 -summing with

$$
\pi_{1}(T) \leq 2^{n+1} K_{G}^{n} \mathrm{ub}\left(\hat{\otimes}_{\alpha, j=1}^{n} \ell_{2}\right) .
$$

Theorem 1.1 gives us then that $T$ is Hilbert-Schmidt with

$$
\|T\|_{S_{2}} \leq 2^{\frac{n}{2}} 2^{n+1} K_{G}^{n} \mathrm{ub}\left(\hat{\otimes}_{\alpha, j=1}^{n} \ell_{2}\right)
$$

and we are done.

We can prove now our main result. We restate it as follows

THEOREM 2.5. There exists positive constants $K, K^{\prime}>0$ that depends only on $n$ such that, if $\alpha$ is a tensor norm such that $\hat{\otimes}_{\alpha, j=1}^{n} \ell_{2}$ has an unconditional basis, then

$$
\frac{1}{K \mathrm{ub}\left(\hat{\otimes}_{\alpha, j=1}^{n} \ell_{2}\right)} \sigma_{2} \leq \alpha \leq K^{\prime} \mathrm{ub}\left(\hat{\otimes}_{\alpha, j=1}^{n} \ell_{2}\right) \sigma_{2} .
$$

Proof. The existence of $K^{\prime}$ is given by Lemma 2.4. To obtain the other inequality, we consider the dual tensor norm $\alpha^{\prime}$. By Theorem 1.4, we have that the canonical tensor basis $\left(e_{i_{1}} \otimes \cdots \otimes e_{i_{n}}\right)_{i_{1}, \ldots, i_{n}=1}^{\infty}$ is unconditional in $\hat{\otimes}_{\alpha, j=1}^{n} \ell_{2}$, 
with unconditional constant bounded by $2^{n+1} \mathrm{ub}\left(\hat{\otimes}_{\alpha, j=1}^{n} \ell_{2}\right)$. Therefore, for each $N \in \mathrm{N}$, as

$$
\left(\otimes_{\alpha^{\prime}, j=1}^{n} \ell_{2}^{N}\right)=\left(\otimes_{\alpha, j=1}^{n} \ell_{2}^{N}\right)^{*}
$$

the unconditional constant of $\left(e_{i_{1}} \otimes \cdots \otimes e_{i_{n}}\right)_{i_{1}, \ldots, i_{n}=1}^{N}$ in $\otimes_{\alpha^{\prime}, j=1}^{n} \ell_{2}^{N}$ is uniformly bounded by

$$
2^{n+1} \mathrm{ub}\left(\hat{\otimes}_{\alpha, j=1}^{n} \ell_{2}\right)
$$

and, therefore, $\left(e_{i_{1}} \otimes \cdots \otimes e_{i_{n}}\right)_{i_{1}, \ldots, i_{n}=1}^{\infty}$ is an unconditional basis also in $\otimes_{\alpha^{\prime}, j=1}^{n} \ell_{2}$, with constant bounded by $2^{n+1} \mathrm{ub}\left(\hat{\otimes}_{\alpha, j=1}^{n} \ell_{2}\right)$.

We can use now Lemma 2.4 to obtain that

$$
\alpha^{\prime} \leq K^{\prime} \mathrm{ub}\left(\hat{\otimes}_{\alpha^{\prime}, j=1}^{n} \ell_{2}\right) \sigma_{2} \leq K^{\prime} 2^{n+1} \mathrm{ub}\left(\hat{\otimes}_{\alpha, j=1}^{n} \ell_{2}\right) \sigma_{2} .
$$

As $\sigma_{2}^{\prime}=\sigma_{2}$, we obtain the left inequality of (4) with $K=K^{\prime} 2^{n+1}$.

We can also give a symmetric version of Theorem 2.5. We just have to reproduce the proof given in [6, Corollary 4] for the case of two spaces. For definitions and notation on symmetric tensor products see [13].

THEOREM 2.6. If $\alpha$ is a symmetric tensor norm such that $\otimes_{\alpha}^{n, s} \ell_{2}$ has an unconditional basis, then $\alpha$ is equivalent to the Hilbert-Schmidt symmetric tensor norm.

Acknowledgement. The authors would like to thank D. García and M. Maestre for asking us the question we solve here and for many helpful conversations during the elaboration of this paper.

\section{REFERENCES}

1. Bombal, F., Pérez-García, D., and Villanueva, I., Multilinear extensions of Grothendieck's theorem, to appear in Quart. J. Math. Oxford Ser. (2).

2. Cobos, F., Kühn, T., and Peetre, J., Schatten-von Neumann classes of multilinear forms, Duke Math. J. 65 (1992), 121-156.

3. Cobos, F., Kühn, T., and Peetre, J., On $\mathscr{S}_{p}$-classes of trilinear forms, J. London Math. Soc. (2) 59 (1999), 1003-1022.

4. Cobos, F., Kühn, T., and Peetre, J., Extreme points of the complex binary trilinear ball, Studia Math. 138 (2000), 81-92.

5. Cobos, F., Kühn, T., and Peetre, J., Remarks on symmetries of trilinear forms. Perspectives in mathematical analysis, Rev. Real Acad. Cienc. Exact. Fís. Natur. Madrid 94 (2000), 441-449.

6. Defant, A., Díaz, J. C., García, D., and Maestre, M., The Pisier-Schütt Theorem for Spaces of Polynomials. Lecture Notes in Pure and Appl. Math. 214 (2000), 55-62.

7. Defant, A., Díaz, J. C., García, D., and Maestre, M., Unconditional basis and Gordon-Lewis constants for spaces of polynomials, J. Funct. Anal. 181 (2001), 119-145.

8. Defant, A., Díaz, J. C., García, D., and Maestre, M., Existence of unconditional bases in spaces of polynomials ond holomorphic functions, Math. Nachr. 233-234 (2002), 89-102. 
9. Defant, A., and Floret, K., Tensor Norms and Operator Ideals, North-Holland, 1993.

10. Defant, A., and Kalton, N., Unconditionality in spaces of $m$-homogeneous polynomials on Banach spaces, to appear in Quart. J. Math. Oxford Ser. (2).

11. Diestel, J., Jarchow, H., and Tonge, A., Absolutely Summing Operators, Cambridge Univ. Press, 1995.

12. Dwyer III, T. A. W., Partial differential equations in Fischer-Fock spaces for the HilbertSchmidt holomorphy type, Bull. Amer. Math. Soc. 77 (1971), 725-730.

13. Floret, K., Natural norms on symmetric tensor products of normed spaces, Note Mat. 17 (1997), 153-188.

14. Floret, K., and Hunfeld, S., Ultrastability of ideals of homogeneous polynomials and multilinear mappings on Banach spaces, Proc. Amer. Math. Soc. 130 (2001), 1425-1435.

15. Janson, S., Peetre, J., and Rochberg, R., Hankel forms and the Fock space, Rev. Mat. Iberoamericana 3 (1987), 61-138.

16. Kwapień, S., and Pełczyński, A., The main triangle projection in matrix spaces and its applications, Studia Math. 34 (1970), 43-68.

17. Matos, M. C., Fully absolutely summing and Hilbert-Schmidt multilinear mappings, Collect. Math. 54 (2003), 111-136.

18. Pełczyński, A., A characterization of Hilbert-Schmidt operators, Studia Math. 28 (1967), 355-360.

19. Pérez-García, D., The inclusion theorem for multiple summing operators, Studia Math. 165 (2004), 275-290.

20. Pérez-García, D., and Villanueva, I., Multiple summing operators on Banach spaces, J. Math. Anal. Appl. 285 (2003), 86-96.

21. Pérez-García, D., and Villanueva, I., Multiple summing operators on $C(K)$ spaces, Ark. Mat. 42 (2004), 153-171.

22. Pérez-García, D., and Villanueva, I., A composition theorem for multiple summing operators, Preprint.

23. Pietsch, A., Ideals of multilinear functionals (designs of a theory), Proceedings of the Second International Conference on Operator Algebras, Ideals, and their Applications in Theoretical Physics (Leipzig), Teubner-Texte, 1983, pp. 185-199.

24. Pisier, G., Some results on Banach spaces without local unconditional structure, Compositio Math. 37 (1978), 3-19.

25. Schütt, C., Unconditionality in tensor products, Israel J. Math. 31 (1978), 209-216.

ÁREA DE MATEMÁTICA APLICADA

ESCET

UNIVERSIDAD REY JUAN CARLOS

DEPARTAMENTAL II

28933 MÓSTOLES (MADRID)

SPAIN

E-mail: dperezg@escet.urjc.es
DEPARTAMENTO DE ANÁLISIS MATEMÁTICO FACULTAD DE MATEMÁTICAS UNIVERSIDAD COMPLUTENSE DE MADRID MADRID 28040 SPAIN

E-mail: ignaciov@mat.ucm.es 